\title{
Uncertainty Evaluation in Modelling of Acoustic Phenomena with Uncertain Parameters Using Interval Arithmetic
}

\author{
W. BATKO* AND P. PAWLIK \\ AGH University of Science and Technology, Faculty of Mechanical Engineering and Robotics \\ Department of Mechanics and Vibroacoustics, al. A. Mickiewicza 30, 30-059 Krakow, Poland
}

\begin{abstract}
Uncertainty assessment in modelling of acoustic phenomena with uncertain parameters using interval arithmetic on the example of the reverberation time estimation, are presented in the paper. The application of the classical interval analysis formalism as well as its expansions are shown. Statistical methods of estimation of the reverberation time are based on parameters, which are related, among others, to the geometry of the analysed room, characteristics of sound absorption, and interior transmission. Values of these parameters are usually difficult to determine, which has a significant influence on the modelling result. The interval analysis allows to determine the variability interval of the parameter being estimated. The authors determined the influence of the input parameters uncertainty on the estimated reverberation time, calculated according to the Sabine, Eyring-Norris and Millington-Sette formulae. The uncertainty analysis was performed for the literature data, related to the reverberation time calculations of the room of a certified acoustics.
\end{abstract}

PACS: $43.28 . \mathrm{Lv}, 43.55 . \mathrm{Br}$

\section{Introduction}

The reverberation time is one of the main parameters in the estimation of acoustic properties of rooms. This parameter, at the designing stage, is determined on the grounds of statistical models. Since the modelling result is strictly dependent on input parameters, their inaccuracy influences the results. Inaccuracy of room cubature measurements, approximate values of absorption coefficients of heterogeneous surfaces, as well as not precise assessments of the sound absorption coefficient in air to mention only the most important examples of such inaccuracies.

In order to estimate the influence of the listed inaccuracies on the modelling result the uncertainty analysis was performed. The formalism of the interval arithmetic and its expansions were used. Such approach to the uncertainty estimation of acoustic parameters was discussed in the previous papers of the authors [1].

\section{Formalism of the interval arithmetic and its expansions}

The arithmetic based on interval operations started in the 50-ties, however only in the 60-ties it was named the interval arithmetic (Moore [2, 3]). Its primary application was in controlling the error rounding in numerical calculations. The advantage of such arithmetic is the

* corresponding author; e-mail: batko@agh.edu.pl uncertainty control at each calculation stage. Such approach can lead to overestimation of the results since the classical interval arithmetic does not take into account correlations between sets of errors. Due to this fact, several expansions of this arithmetic and of interval reductive methods were developed. One of such expansions is the reductive interval arithmetic [4], discussed in Sect. 2.2. The method of the precise determination of the resulting interval, in a form of the determination of individual disturbances influence on the analysis result, was also developed. This expansion is discussed in Sect. 2.3.

\subsection{Classical interval arithmetic}

In the classical interval arithmetic, proposed by Moore, intervals are represented as limited sets of real numbers

$$
\boldsymbol{x}=[\underline{x}, \bar{x}]=\{x \in R: \underline{x} \leq x \leq \bar{x}\},
$$

where $x$ is the interval lower limit - infimum, while $\bar{x}$ is the upper limit - supremum, while $x$ is an arbitrary real number belonging to the interval. Interval numbers are written in bold.

The basic arithmetic operations are defined on the interval set:

$$
\boldsymbol{x} \diamond \boldsymbol{y}=\{z=x \diamond y: x \in \boldsymbol{x}, y \in \boldsymbol{y}\},
$$

where $\diamond$ is one of the operators: addition, subtraction, multiplication and division. These operators, apart from division, are defined for arbitrary sets. For division it should be assumed that: $0 \notin \boldsymbol{y}$.

Additions and subtraction of intervals is realised by operations on the end points, according to (3) and (4): 


$$
\begin{aligned}
& \boldsymbol{x}+\boldsymbol{y}=[\underline{x}+\underline{y}, \bar{x}+\bar{y}], \\
& \boldsymbol{x}-\boldsymbol{y}=[\underline{x}-\bar{y}, \bar{x}-\underline{y}] .
\end{aligned}
$$

The interval being the multiplication result is determined on the basis of the smallest and largest product of two end points of the interval $\boldsymbol{x}, \boldsymbol{y}$

$\boldsymbol{x} \cdot \boldsymbol{y}=[\min (\underline{x} \underline{y}, \underline{x} \bar{y}, \bar{x} \underline{y}, \bar{x} \bar{y}), \max (\underline{x} \underline{y}, \underline{x} \bar{y}, \bar{x} \underline{y}, \bar{x} \bar{y})]$.

The division operation is determined by means of reciprocal

$$
\frac{x}{y}=x \frac{1}{y},
$$

while the interval reciprocal is presented by the formula (7)

$$
\frac{1}{\boldsymbol{y}}=\left[\frac{1}{\bar{y}}, \frac{1}{y}\right] \text {; when } \underline{y}>0 \text { or } \bar{y}<0 .
$$

\subsection{Reductive interval arithmetic}

One of the expansions of the Moore's classical interval arithmetic is the reductive interval arithmetic, proposed by Jakubiec [4]. The interval $\boldsymbol{x}=[\underline{x}, \bar{x}]$ - in the reductive interval arithmetic - is written by means of the interval median $\operatorname{mid}(\boldsymbol{x})$ and radius $\operatorname{rad}(\boldsymbol{x})$ :

$$
\begin{aligned}
& \breve{x}=\operatorname{mid}(\boldsymbol{x})=\frac{\underline{x}+\bar{x}}{2}, \\
& \operatorname{rad}(\boldsymbol{x})=\frac{|\bar{x}-\underline{x}|}{2} .
\end{aligned}
$$

Relations in between intervals are the same in the classical and reductive interval arithmetic. However, the crucial advantage of the reductive arithmetic is an introduction of the coherence coefficient, which describes properties and correlation between intervals and in effect reduces the interval width.

The interval - in a sense of the interval arithmetic can be treated as a sum of two independent components: centre $\breve{x}$ and unbiased interval $\pm \operatorname{rad}(\boldsymbol{x})$ :

$$
\begin{aligned}
\boldsymbol{x} & =[\underline{x}, \bar{x}]=[\breve{x}-\operatorname{rad}(\boldsymbol{x}), \breve{x}+\operatorname{rad}(\boldsymbol{x})] \\
& =\breve{x} \pm \operatorname{rad}(\boldsymbol{x})=\breve{x}+[-\operatorname{rad}(\boldsymbol{x}), \operatorname{rad}(\boldsymbol{x})] .
\end{aligned}
$$

Due to such approach operations on intervals can be done separately for these two components. Operations on the interval centres, which are real numbers, are performed according to rules obligatory for these numbers, whereas operations on unbiased intervals are performed by means of the reductive interval arithmetic. For each pair of intervals - in the reductive interval arithmetic the number called the coherence coefficient is determined

$$
r_{i j} \in R, \quad-1<r_{i j}<1 .
$$

This coefficient describes properties of individual uncertainty intervals and dependences in between the given pair of intervals.

Mathematical operations for the reductive arithmetic are described as follows:

$$
\boldsymbol{y}=\boldsymbol{x}_{1}+\boldsymbol{x}_{2}=\breve{y}+[-\operatorname{rad}(\boldsymbol{y}),+\operatorname{rad}(\boldsymbol{y})],
$$

where

$$
\begin{aligned}
& \breve{y}=\operatorname{mid}(\boldsymbol{y})=\breve{x}_{1}+\breve{x}_{2}, \\
& \operatorname{rad}(\boldsymbol{y})=\sqrt{\operatorname{rad}^{2}\left(\boldsymbol{x}_{1}\right)+\operatorname{rad}^{2}\left(\boldsymbol{x}_{2}\right)+2 \operatorname{rad}\left(\boldsymbol{x}_{1}\right) \operatorname{rad}\left(\boldsymbol{x}_{2}\right) r_{12}} .
\end{aligned}
$$

For the coefficient of coherence equal to 1 the same dependences as for the Moore's classical arithmetic are obtained, however when this coefficient is different, the interval width decreases.

\subsection{Perturbation interval arithmetic}

Subsequent expansion of the classical interval arithmetic was done by Skrzypczyk. This method was created from combining two algebraic systems, Moore's interval numbers [2,3] and perturbation numbers of Skrzypczyk [5].

By using dependence (12) the interval number can be written in a form

$$
\boldsymbol{x}=[\breve{x}-\operatorname{rad}(\boldsymbol{x}), \breve{x}+\operatorname{rad}(\boldsymbol{x})] .
$$

It is assumed in the perturbation arithmetic that the interval radius is the perturbation number

$$
\begin{aligned}
& \operatorname{rad}(\boldsymbol{x})=\varepsilon_{1} \delta x_{1}+\varepsilon_{2} \delta x_{2}+\ldots+\varepsilon_{n} \delta x_{n}, \\
& \delta x_{1}, \delta x_{2} \ldots \delta x_{n} \geq 0 .
\end{aligned}
$$

By defining $n$ independent perturbation intervals $\varepsilon_{i}=$ $\left[-\varepsilon_{i}, \varepsilon_{i}\right], i=1,2,3 \ldots n$ it is possible to write the perturbation interval number as

$$
\boldsymbol{x}=\breve{x}+\varepsilon_{1} \delta x_{1}+\varepsilon_{2} \delta x_{2}+\ldots+\varepsilon_{n} \delta x_{n} .
$$

Algebraic operations in the perturbation interval arithmetic are realised as follows:

$$
\begin{aligned}
& \boldsymbol{x}+\boldsymbol{y}=\breve{x}+\breve{y}+\varepsilon_{1}\left(\delta x_{1}+\delta y_{1}\right)+\varepsilon_{2}\left(\delta x_{2}+\delta y_{2}\right) \\
& \quad+\ldots+\varepsilon_{n}\left(\delta x_{n}+\delta y_{n}\right), \\
& \boldsymbol{x}-\boldsymbol{y}=\breve{x}-\breve{y}+\varepsilon_{1}\left(\delta x_{1}+\delta y_{2}\right)+\varepsilon_{2}\left(\delta x_{1}+\delta y_{2}\right) \\
& \quad+\ldots+\varepsilon_{n}\left(\delta x_{n}+\delta y_{n}\right), \\
& \boldsymbol{x} \cdot \boldsymbol{y}=\breve{x} \breve{y}+\varepsilon_{1}\left(\breve{x} \delta y_{1}+\breve{y} \delta x_{1}\right)+\varepsilon_{2}\left(\breve{x} \delta y_{2}+\breve{y} \delta x_{2}\right) \\
& \quad+\ldots+\varepsilon_{n}\left(\breve{x} \delta y_{n}+\breve{y} \delta x_{n}\right), \\
& 1 / \boldsymbol{x}=1 / \breve{x}-\varepsilon_{1}\left(\delta x_{1} / \breve{x}^{2}\right)-\varepsilon_{2}\left(\delta x_{2} / \breve{x}^{2}\right)-\varepsilon_{n}\left(\delta x_{n} / \breve{x}^{2}\right) .
\end{aligned}
$$

Successive components at $\varepsilon_{i}$ in the perturbation interval arithmetic represent disturbances originating from various sources. Applying this expansion of the interval arithmetic to uncertainty estimation, it is possible to determine not only the variability range but also the influence of individual error sources on the final result.

\section{Interval arithmetic formalism in the uncertainty determination in modelling}

Applying the computation formalism of the interval arithmetic the uncertainty analysis of the reverberation 
sound modelling - by statistical methods, according to formulae of: Sabine, Eyring-Norris and Millington-Sette - was performed. A significant role in the modelling process plays the coefficient of sound absorption $\alpha$, which the most often is experimentally determined and since then biased with uncertainty. This uncertainty can have a significant influence on the modelling result, similarly as errors related to the room cubature measurements $V, S$ and the coefficient of absorption by air $m$.

On the basis of measurements carried out in the Lviv State University Assembly Hall by the authors of paper [6], the reverberation time was determined from the Sabine 's equation with expansion containing absorption by air

$$
\begin{aligned}
T_{\mathrm{Sab}} & =\frac{0.16 V}{4 m V+S \alpha_{\mathrm{Sab}}}, \\
\alpha_{\mathrm{Sab}} & =\frac{1}{S} \sum_{i=1}^{n} \alpha_{i} S_{i}, \quad S=\sum_{i=1}^{n} S_{i},
\end{aligned}
$$

where $V$ - room volume $\left[\mathrm{m}^{3}\right], S$ - total surface of the room $\left[\mathrm{m}^{2}\right], m$ - coefficient of absorption by air $[\mathrm{Np} / \mathrm{m}]$, $\alpha_{\text {Sab }}-$ average sound absorption coefficient.

The following data were used in calculations: $V=$ $3400 \mathrm{~m}^{3}, m=2.7 \times 10^{-3} \mathrm{~Np} / \mathrm{m}$. The sound absorption coefficients for individual surfaces $\alpha_{i}$ and coordinates of points determining surfaces $S_{i}$ are taken from the results of the measurements carried out by the authors of work [6].

Uncertainties of the model input parameters $V, \quad S, \quad m, \alpha_{i}$ were determined in a form of their variability intervals. Using the formalism of the Moore's classical interval arithmetic [2, 3] intervals of the reverberation time variability $T_{\mathrm{Sab}}$ were determined in dependence of uncertainty of input parameters expressed in percentages (Fig. 1). The analysis of the uncertainty of modelling when other statistical methods were applied was also performed. The results are presented in Fig. 1.

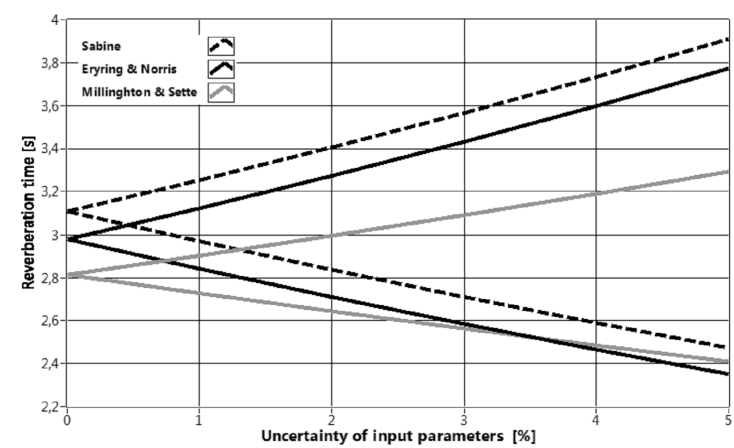

Fig. 1. Reverberation time estimated by various statistical methods for the frequency of $125 \mathrm{~Hz}$ and the parameters uncertainty from $0 \%$ to $5 \%$.

Then the uncertainty of the reverberation time estimation by the Sabine 's method was assessed (with taking into account the air absorption) using the perturbation interval arithmetic. The influence of $\alpha_{\text {Sab }}$ and $m$ parameters on the modelling result was investigated. The obtained analysis results were compared with the ones obtained by means of the classical interval analysis (Fig. 2).

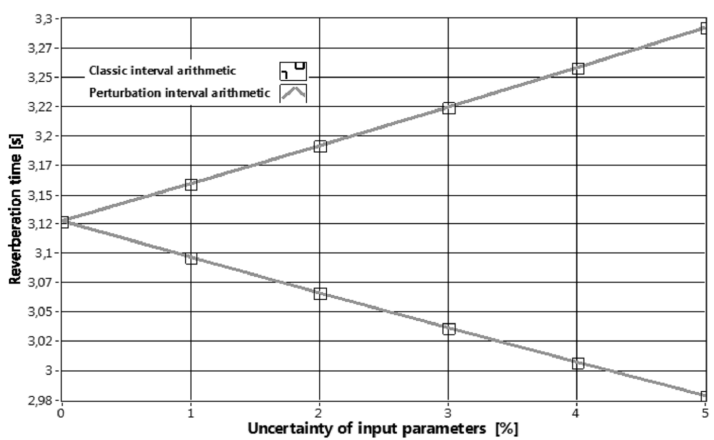

Fig. 2. Reverberation time estimated by various statistical methods for the frequency $125 \mathrm{~Hz}$ and the $\alpha_{\mathrm{Sab}}$ and $m$ parameters uncertainty from $0 \%$ to $5 \%$.

As can be seen in the above figure the perturbation interval arithmetic provides the same results as the classical one, however, its advantage is the possibility of estimation of the influence of individual perturbations (disturbances) variability on the uncertainty of the result. Applying the properties of the perturbation arithmetic, specifying $\alpha_{\text {Sab }}$ and $m$ as perturbation interval numbers, the Sabine 's formula can be written as follows:

$$
\begin{aligned}
\breve{T}_{\mathrm{Sab}} & =\frac{0.16 V}{4 \breve{m} V+S \breve{\alpha}_{\mathrm{Sab}}}, \\
T_{\mathrm{Sab}, i} & =-\frac{0.16 V\left(4 V \delta m_{i}+S \delta \alpha_{i}\right)}{\left(4 V \breve{m}+S \breve{\alpha}_{\mathrm{Sab}}\right)^{2}},
\end{aligned}
$$

where $\breve{T}_{\text {Sab }}$ is the interval centre, it means the formula without disturbances, while $T_{\mathrm{Sab}, i}$ is the $i$-th interval perturbation of the reverberation time. Each successive perturbation can be the result of disturbances of input parameters originating from other error sources.

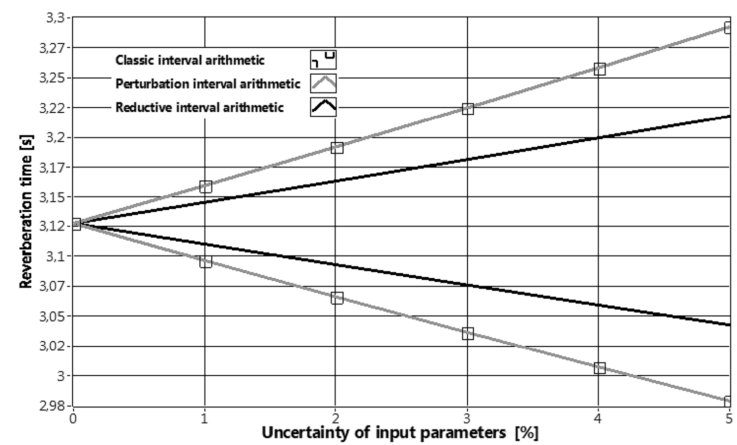

Fig. 3. Reverberation time determined by various statistical methods for the frequency $125 \mathrm{~Hz}$, with the uncertainty of $\alpha_{\text {Sab }}$ and $m$ parameters from $0 \%$ to $5 \%$.

The successive experiment constituted the application of the reductive interval arithmetic for the uncertainty 
estimation. The analysis of the influence of the parameters $\alpha_{\text {Sab }}$ and $m$ uncertainty, at the given coherence coefficient, on the uncertainty of the modelling result, was performed. For the coherence coefficient $r=1$ the estimation results are the same as the results of the classical interval arithmetic. However, after taking into account the distribution of $\alpha_{\text {Sab }}$ and $m$ parameter (at the coherence coefficient $r=0.334$ ) the interval of the reverberation time uncertainty decreased, which indicates more precise error representation (Fig. 3).

Determination of properties of error sources as well as their mutual relations enables more precise uncertainty analysis. However, it is not always possible to determine the correlation between input parameters.

\section{Conclusions}

Analyses performed by various interval methods indicated significant influence parameters of the reverberation time models on the estimated result. Application of interval arithmetic expansions enables the more precise determination of the modelling uncertainty - reductive interval arithmetic, as well as the estimation influence of individual disturbances - perturbation interval arithmetic. Disturbances can simultaneously influence several parameters, which can indicate the correlation in between them. Thus, both arithmetic expansions seem to be a good tool for the estimation of the acoustic parameters uncertainty, including the reverberation time.

\section{References}

[1] W. Batko, P. Pawlik, in: DAGA 2011 - The 3rth Annual Conf. for Acoustics, Deutsche Gesellschaft für Akustik e.V., Düsseldorf 2011, p. 71.

[2] R.E. Moore, Ph.D. Thesis, Stanford University, Stanford 1962.

[3] R.E. Moore, Interval Analysis, Prentice-Hall, Englewood Cliffs, USA 1966.

[4] J. Jakubiec, Application of Reductive Interval Arithmetic to Uncertainty Evaluation of Measurement Data Processing Algorithms, Silesian University of Technology Press, Gliwice 2002, p. 23.

[5] J. Skrzypczyk, Perturbation Methods 1. New Algebraic Methodology, Applications in Mechanics and Acoustics, Silesian University of Technology Press, Gliwice 2010, p. 29, 165.

[6] T. Kamisiński, R. Kinash, A. Pilch, J. Rubacha, in: Building Physics in Theory and Practice, Planning and Research Studio 5E, Łódź 2011, p. 169. 